\title{
Bloqueio 3-em-1 Prolongado versus Analgesia Sistêmica no Tratamento da Dor Pós-Operatória após a Reconstrução do Ligamento Cruzado Anterior do Joelho* Extended Three-in-One Block versus Intravenous Analgesia for Postoperative Pain Management after Reconstruction of Anterior Cruciate Ligament of the Knee
}

\author{
Víctor A. Contreras-Domínguez¹, Paulina E. Carbonell-Bellolio², Álvaro C. Ojeda-Greciet ${ }^{3}$, Edgardo S. Sanzana ${ }^{4}$
}

\section{RESUMO}

Contreras-Domínguez VA, Carbonell-Bellolio PE, Ojeda-Greciet AC, Sanzana ES - Bloqueio 3-em-1 Prolongado versus Analgesia Sistêmica no Tratamento da Dor Pós-Operatória após a Reconstrução do Ligamento Cruzado Anterior do Joelho.

JUSTIFICATIVA E OBJETIVOS: O bloqueio femoral contínuo (3-em-1) é usado para a analgesia pós-operatória de artroplastia de quadril e joelho com bons resultados, apresentando vantagens sobre outras técnicas de analgesia locorregional ou sistêmica e com baixa incidência de complicações. O objetivo deste estudo foi avaliar clinicamente a utilidade do bloqueio femoral contínuo em comparação com a analgesia por via venosa na reconstrução do ligamento cruzado anterior.

MÉTODO: Foi realizado um estudo prospectivo, controlado, com 60 pacientes com estado físico ASA I. Os paciente foram divididos em dois grupos: Grupo 1 ( $n=30)$ : bloqueio femoral contínuo com infusão de bupivacaína e clonidina; Grupo $2(n=30)$ : infusão por via venosa de cetoprofeno. A intervenção cirúrgica foi realizada sob raquianestesia e sedação. O tratamento da dor pós-operatória foi feito com analgesia controlada pelo paciente (PCA) usando morfina. A dor pós-operatória foi registrada 2, 4, 6, 24 e 36 horas após a intervenção cirúrgica usando a Escala Visual Analógica (VAS). $O$ consumo de morfina, a satisfação dos pacientes e as complicações também foram registradas.

RESULTADOS: No Grupo 1, o VAS pós-operatório entre 4 e 48 horas após a intervenção cirúrgica foi de $21 \mathrm{~mm} \pm 2$ e no Grupo 2 foi de $45 \mathrm{~mm} \pm 4(p<0,001)$. O uso de morfina no período de 4 a 48

\footnotetext{
${ }^{*}$ Recebido do (Received from) Departmento de Anestesiología e Cirugía Ortopédica, Facultad de Medicina, Universidad de Concepción, Concepción, Chile

1. Professor Assistente de Anestesiologia, Facultad de Medicina, Universidad de Concepción

2. Anestesiologista, Departamento de Anestesiología, Hospital Traumatologico de Concepción

3. Cirurgião Ortopédico, Departmento de Cirugía Ortopédica, Hospital Traumatologico de Concepción

4. Instrutor de Cirurgia Ortopédica, Facultad de Medicina, Universidad de Concepción

Apresentado (Submitted) em 19 de maio de 2006

Aceito (Accepted) para publicação em 2 de fevereiro de 2007

Endereço para correspondência (Correspondence to):

Dr. Víctor A. Contreras-Domínguez

Regional Clinical Hospital of Concepción

200 Janequeo St.

Concepción, Chile

E-mail: viccontredom@yahoo.com
}

(c) Sociedade Brasileira de Anestesiologia, 2007 horas no Grupo 1 foi de 4,5 mg 1,5 e no Grupo 2 foi de 25,5 mg $\pm 3(p<0,001)$. No Grupo 1, 6,7\% dos pacientes apresentaram náusea ou vômito no pós-operatório (NVPO) comparado com $20 \%$ no Grupo 2.

CONCLUSÕES: O bloqueio 3-em-1 com bupivacaína e clonidina em infusão contínua forneceu analgesia mais eficaz, maior satisfação do paciente, reduziu o consumo de morfina por via venosa $e$ apresentou menor incidência de NVPO do que a analgesia sistêmica.

Unitermos: ANALGESIA, Regional: bloqueio 3-em-1, sistêmica; ClRURGIA, Ortopédica: reconstrução do ligamento cruzado anterior.

\section{SUMMARY}

Contreras-Domínguez VA, Carbonell-Bellolio PE, Ojeda-Greciet AC, Sanzana ES - Extended Three-in-One Block versus Intravenous Analgesia for Postoperative Pain Management after Reconstruction of Anterior Cruciate Ligament of the Knee.

BACKGROUND AND OBJECTIVES: Continuous femoral block (three-in-one) is used for postoperative analgesia in hip and knee replacements with good results, with advantages over other locoregional analgesic or intravenous techniques having low incidence of complications. The aim of this study was to clinically evaluate the utility of continuous femoral block compared with intravenous analgesia in reconstruction of anterior cruciate ligament.

METHODS: Controlled prospective study of 60 patients ASA I. Patients were divided into two groups: Group $1(n=30)$ : continuous femoral block with an infusion of bupivacaine and clonidine; and Group $2(n=30)$ : intravenous ketoprofen infusion. Surgery was performed under spinal anesthesia and sedation. Postoperative pain management with morphine patient controlled analgesia (PCA). Postoperative pain recorded at 2, 4, 6, 24 and 36 hours after surgery using Visual Analog Scale (VAS). Morphine consumption, satisfaction's score and complications are registered.

RESULTS: In Group 1 postoperative VAS between 4 and 48 hours was $21 \mathrm{~mm} \pm 2$ and in Group 2 was $45 \mathrm{~mm} \pm 4$ ( $p<0.001)$. Morphine consumption between 4 and 48 hours in Group 1 was $4.5 \mathrm{mg} \pm 1.5$ and in Group 2 was $25.5 \mathrm{mg} \pm 3(p<0.001)$. In Group 1, 6.7\% of patients presented postoperative nausea or vomiting (PONV) compared to $20 \%$ in Group 2.

CONCLUSION: The three-in-one block with bupivacaine and clonidine in continuous infusion provides more efficient analgesia, patient satisfaction and less consumption of intravenous morphine and PONV than intravenous analgesia.

Key Words: ANALGESIA, Regional: three-in-one block, systemic; SURGERY, Orthopedic: anterior cruciate ligament reconstruction. 


\section{BLOQUEIO 3 EM 1 PROLONGADO VERSUS ANALGESIA SISTÊMICA NO TRATAMENTO DA DOR PÓS-OPERATÓRIA APÓS A RECONSTRUÇÃO DO LIGAMENTO CRUZADO ANTERIOR DO JOELHO}

\section{INTRODUÇÃO}

$\mathrm{O}$ bloqueio femoral contínuo (BFC) tem sido utilizado com bons resultados como técnica analgésica pós-operatória em artroplastia de quadril e joelho ${ }^{1-3}$ e em enxertos em pacientes queimados ${ }^{4}$, apresentando grandes vantagens e poucos efeitos colaterais quando comparado com outras técnicas de anestesia locorregional ou sistêmica 5,6. $\mathrm{O} B F C$ é excelente alternativa à analgesia peridural (AP) ou analgesia controlada pelo paciente (PCA) com opióides por via venosa no tratamento da dor aguda em intervenções cirúrgicas ortopédicas de grande porte ${ }^{7}$. Essa técnica também evita complicações, como a hemorragia peridural que pode ocorrer em pacientes em tratamento com heparina de baixo peso molecular ${ }^{8}$.

A infusão contínua de anestésicos locais (AL) pode produzir efeitos sistêmicos, como confusão, hipotensão arterial, hipóxia, disritmias cardíacas, convulsões e coma. Entretanto, essas complicações são raras com o uso do BFC devido à baixa concentração de AL usado e de seu baixo nível plasmático ${ }^{9}$. Durante a AP é freqüente a ocorrência de hipotensão arterial, depressão cardiorrespiratória ou sintomas neurológicos, especialmente quando usada em pacientes idosos ou de alto risco. $\mathrm{O} B F C$ parece oferecer vantagens em relação à $A P{ }^{10}$. A dor após a reconstrução do ligamento cruzado anterior (LCA) é de moderada intensidade em $89 \%$ dos pacientes. A administração profilática de analgésicos (por exemplo, ceterolaco $60 \mathrm{mg}$ por via venosa) ou intra-articular de AL (bupivacaína $1 \mathrm{mg} \mathrm{kg}^{-1}$ ) associada à analgesia oral pós-operatória ${ }^{11}$ não altera o escore de dor. O objetivo deste estudo foi avaliar clinicamente a eficácia do bloqueio femoral contínuo comparado com a analgesia, por via venosa, na reconstrução artroscópica do ligamento cruzado anterior do joelho.

\section{MÉTODOS}

Após aprovação pelo Conselho de Ética da instituição e assinatura do consentimento informado, 60 pacientes, estado físico ASA I, a serem submetidos à artroscopia eletiva para reconstrução unilateral do ligamento cruzado anterior (LCA) do joelho, sob raquianestesia, participaram deste estudo. Foram incluídos pacientes com idade entre 18 e 51 anos e índice de massa corporal (IMC) $<30$. Os critérios de exclusão incluíram: anormalidades de coagulação, alergia ao AL, infecção local, déficit neuronal preexistente, hipertensão arterial, diabete melito, doença hepática ou renal, tratamento crônico com alfa ${ }_{2}$-agonistas (clonidina), ou se não fossem capazes de entender a escala de dor ou o uso do PCA.

Os pacientes foram divididos aleatoriamente em dois grupos de 30 pacientes, sendo que o Grupo 1 recebeu, no pósoperatório, uma infusão contínua de bupivacaína a 0,125\% mais $1 \mu \mathrm{g} \cdot \mathrm{mL}^{-1}$ de clonidina a uma velocidade de $10 \mathrm{~mL} \cdot \mathrm{h}^{-1}$ através de cateter femoral, e o Grupo 2 recebeu administração contínua de cetoprofeno por via venosa na dose de $300 \mathrm{mg}$ em 24 horas.
Em todos os pacientes do Grupo 1 o bloqueio $3 \mathrm{em} 1$ foi realizado antes da raquianestesia usando os pontos de referência de Winnie e col. ${ }^{12}$. A artéria femoral foi localizada abaixo do ligamento inguinal e uma cânula de $18 \mathrm{G}$ de bisel curto (Polyplex ${ }^{\circledR}$ C 50, Polymedic, Carneres sur Seine, França) foi inserida lateralmente à artéria. O nervo femoral foi localizado com um estimulador de nervo periférico (TOF Watch $S^{\circledast}$, Organon, Irlanda). Usando-se uma descarga inicial de 1,5 mA, a agulha foi introduzida, com aspiração inztermitente, em um ângulo de $30^{\circ}$ a $45^{\circ} \mathrm{com}$ a pele até que ocorresse contração do quadríceps femoral (elevação da patela).

Sua posição foi otimizada e considerada adequada quando uma descarga igual ou menor que $0.4 \mathrm{~mA}$ ainda causava contração do quadríceps. Um cateter de calibre $20 \mathrm{G}$ foi então introduzido $12 \mathrm{~cm}$ na bainha do nervo femoral usando a técnica de Seldinger. Depois que o teste de aspiração foi negativo para sangue, o cateter foi fixado.

Todos os pacientes em ambos os grupos receberam raquianestesia. A punção lombar foi feita no espaço $L_{3}-L_{4}$ ou $\mathrm{L}_{4}-\mathrm{L}_{5}$ com agulha ponta de lápis $25 \mathrm{G}$, sendo administrados $12,5 \mathrm{mg}$ de bupivacaína hiperbárica a $0,75 \%$ com $2,5 \mu \mathrm{g}$ de sulfentanil. A sedação durante procedimento cirúrgico foi feita pela infusão alvo-controlada (IAC) de propofol a $1 \%$ (Base Primea Orchestra ${ }^{\circledR}$, Fressenius-Vial, França). Todos os pacientes também receberam $30 \mathrm{mg}$ de ceterolaco por via venosa antes do início da isquemia pneumática e três vezes ao dia nas 48 horas seguintes.

$\mathrm{Na}$ sala de recuperação pós-anestésica foi confirmado o posicionamento correto do cateter na bainha femoral através da injeção de $10 \mathrm{~mL}$ de bupivacaína a $0,125 \%$ mais $1 \mu \mathrm{g} \cdot \mathrm{mL}^{-1}$ de clonidina. A perda da sensação de temperatura (bloqueio sensitivo) foi avaliada usando-se algodão embebido em éter na distribuição do nervo femoral (face anterior da coxa). Após esse teste, foi iniciada a infusão de solução de bupivacaína a $0,125 \%$ e clonidina $\left(1 \mu \mathrm{g} \cdot \mathrm{mL}^{-1}\right)$ a uma velocidade de $10 \mathrm{~mL} \cdot \mathrm{h}^{-1}$ (Grupo 1) usando-se a bomba PMP (Abbott Laboratories, USA). Os pacientes do Grupo 2 receberam analgesia contínua por via venosa com $300 \mathrm{mg}$ de cetoprofeno em 24 horas. A analgesia suplementar padronizada incluía administração de morfina por via venosa por PCA (bolus de $1,5 \mathrm{mg}$, interrupção por 10 minutos, faixa de segurança de $20 \mathrm{mg}$ em 4 horas).

Imediatamente após a alta da sala de recuperação pósanestésica, foi iniciado o mesmo tratamento farmacológico em todos os pacientes (antibióticos e heparina de baixo peso molecular) e assistência cinética com um aparelho de movimento passivo que foi utilizado 8 horas após o procedimento cirúrgico.

A dor foi medida usando-se a escala analógica visual (VAS, que varia de $0=$ sem dor a $100=$ pior dor imaginável) registrada 2, 6, 12, 24, 36 e 48 horas após o procedimento cirúrgico. Os efeitos colaterais, consumo de morfina e grau de satisfação dos pacientes (usando a VAS, em que $0=$ não satis- 
feito e $100=$ completamente satisfeito) foram registrados ao final do estudo.

Todos os dados foram colhidos por outros anestesistas que não estiveram envolvidos nos procedimentos ou no tratamento dos pacientes na sala de recuperação pós-anestésica.

Análise de poder estatístico sugeriu que seriam necessários 15 pacientes em cada grupo de estudo para observar redução de pelo menos $50 \%$ na escala VAS entre os grupos de analgesia contínua por via venosa e bloqueio femoral e que houvesse probabilidade de $95 \%$ de detectar tal redução com um nível de significância de 0,01. Para aumentar o significado dos resultados, decidiu-se incluir 30 pacientes em cada grupo. A análise estatística foi feita usando-se a Análise de Variância e o teste de Bonferroni $t$ para dados paramétricos. A necessidade de morfina pela PCA foi analisada pelo teste Kruskal-Wallis. Os dados demográficos foram comparados usando-se o teste Qui-quadrado. Valores de $p<0,05$ foram considerados significativos. Os resultados foram expressos como média \pm DP.

\section{RESULTADOS}

Os dados demográficos estão apresentados na tabela I. Não foi notada diferença entre os grupos. Na sala de recuperação pós-anestésica, a perda completa da sensação de temperatura na distribuição do nervo femoral foi observada em todos os pacientes do Grupo 1.

Os valores da VAS 2, 4, 6, 24, 36 e 48 horas após a intervenção cirúrgica, consumo de morfina, incidência de náusea e vômitos (NVPO) e o grau de satisfação dos pacientes 48 horas após a operação encontram-se na tabela II.

O escore de dor após 4, 6, 24, 36 e 48 h foi significativamente menor no Grupo 1 quando comparado com o Grupo 2

Tabela I - Dados Demográficos

\begin{tabular}{lccc}
\hline & Grupo $1(\mathrm{n}=30)$ & Grupo $2(\mathrm{n}=30)$ & $\mathrm{p}$ \\
\hline Idade $(\mathrm{anos})^{*}$ & $25 \pm 3$ & $23 \pm 4$ & 0,27 \\
Peso $(\mathrm{kg})^{*}$ & $72 \pm 4$ & $75 \pm 3$ & 0,83 \\
Altura $(\mathrm{cm})^{*}$ & $166 \pm 3$ & $168 \pm 3$ & 0,39 \\
Sexo $(\mathrm{M} / \mathrm{F})$ & $18 / 2$ & $18 / 2$ & 0,65 \\
Duração do procedimento $(\mathrm{min})^{*}$ & $110 \pm 5$ & $107 \pm 3$ & 0,71 \\
\hline
\end{tabular}

Grupo 1 - bloqueio femoral contínuo (3 em 1); Grupo 2 - analgesia contínua por via venosa.

* Valores expressos em Média \pm DP.

A análise estatística foi feita usando-se a Análise de Variância ou Qui-quadrado.

Tabela II - Grau de Dor, Consumo de Morfina, Náusea e Vômitos Pós-Operatórios e Satisfação dos Pacientes nos Dois Grupos

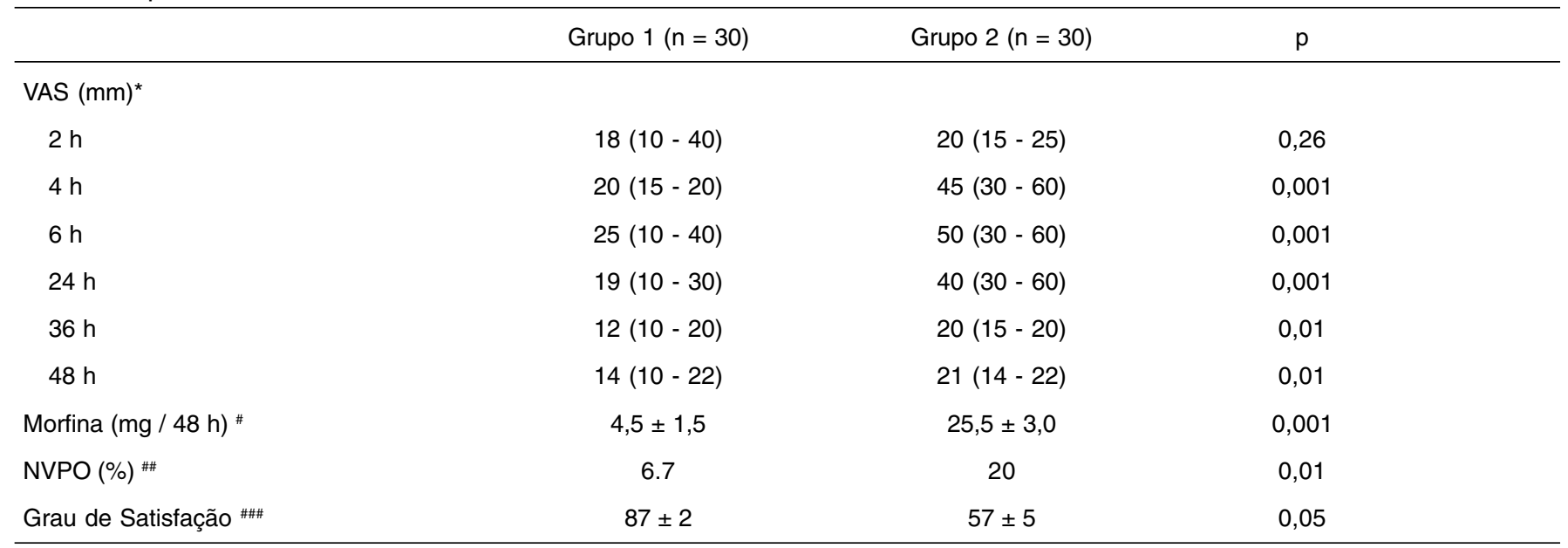

Grupo 1 - bloqueio femoral contínuo (3 em 1); Grupo 2 - analgesia contínua por via venosa; VAS - escala analógica visual; NVPO náusea e vômitos pós-operatórios.

* Expresso como Mediana (Percentis 25-75).

\# Valores expressos em Média \pm DP.

\#\# Expressos como percentagem.

\#\#\# Expressos como razão (VAS varia de $0=$ não satisfeito a $100=$ completamente satisfeito). 


\section{BLOQUEIO 3 EM 1 PROLONGADO VERSUS ANALGESIA SISTÊMICA NO TRATAMENTO DA DOR PÓS-OPERATÓRIA APÓS A RECONSTRUÇÃO DO LIGAMENTO CRUZADO ANTERIOR DO JOELHO}

( $p<0,001$ e $p<0,01)$. O consumo de morfina foi muito maior no Grupo 2 quando comparado com o Grupo 1 ( $p<0,001)$. O grau de satisfação dos pacientes foi menor que 60 no Grupo 2 e maior que 80 no Grupo 1 ( $p<0,05)$.

A incidência de NVPO no Grupo 1 foi significativamente menor do que no Grupo 2 ( $p<0,01)$.

No Grupo 1, o bloqueio femoral manteve-se bem durante todo o período do estudo. Exceto por NVPO, nenhum outro efeito colateral ou problema técnico foi notado nos dois grupos.

\section{DISCUSSÃO}

Este estudo prospectivo, com distribuição aleatória, mostrou que o bloqueio do nervo femoral com a bupivacaína e clonidina em infusão contínua fornece analgesia eficaz, grande grau de satisfação do paciente, redução nos escores de dor e no consumo de morfina por via venosa e menor incidência de NVPO do que a analgesia por via venosa.

A reconstrução do LCA baseado na técnica "osso-tendãoosso" (enxerto autólogo do tendão patelar) é altamente recomendada em desportistas jovens devido à sua maior resistência biomecânica e aos bons resultados funcionais observados em séries de casos com seguimento longo.

O controle da dor pós-operatória após a reconstrução do LCA tem sido feito usando-se analgesia por via venosa (técnicas contínuas ou $\mathrm{PCA}$ ), analgesia intra-articular (AI), anestesia peridural (AP) e bloqueio do nervo femoral (bloqueio $3 \mathrm{em} \mathrm{1),}$ em dose única ou contínua.

A analgesia por via venosa nos pacientes submetidos a anestesia geral ou regional está associada com alto consumo de morfina, alta incidência de náusea e vômitos no pósoperatório (NVPO) e redução no grau de satisfação dos pacientes ${ }^{13}$. Isso foi corroborado nessa série de 60 pacientes, em que o grupo de analgesia por via venosa apresentou mais dor, maior consumo de morfina, maior incidência de NVPO e menor satisfação quando comparado com os pacientes do grupo do bloqueio "3 em 1" $(p<0,001)$. O papel de outros analgésicos por via venosa pode ser interessante no tratamento de pacientes submetidos a procedimentos ortopédicos sob anestesia geral. A administração de pequenas doses de cetamina no período perioperatório reduz a necessidade de morfina no pós-operatório e melhora a mobilização 24 horas após o reparo artroscópico do ligamento anterior ${ }^{13,14}$. Essa estratégia pode ser usada em associação com a analgesia por via venosa. Nesta experiência, o consumo pós-operatório de morfina e a incidência de NVPO foi significativamente maior no Grupo 2 do que no grupo do bloqueio "3 em 1".

A analgesia peridural fornece boa analgesia pós-operatória, mas aumenta a incidência de efeitos colaterais, como a hipotensão arterial, bloqueio motor e sensitivo bilateral e retenção urinária. Os problemas associados com a manutenção de cateter peridural e o risco de hematoma peridural em pacientes recebendo tratamento anticoagulante reduzem seu uso ${ }^{8}$.
Chew e col. ${ }^{15}$ demonstraram a utilidade da infusão contínua de bupivacaína a $0,25 \%$ ou a $0,5 \%$ no compartimento infrapatelar. Branson e col. ${ }^{16}$ usaram morfina intra-articular (IA) diluída (2 ou $5 \mathrm{mg}$ ) com bons resultados nas primeiras 24 horas. Após esse período, a dor aumentou, dificultando a terapia cinética. A técnica IA está limitada ao controle da dor pós-operatória em operações de grande porte do joelho, pois sua utilidade está restrita às primeiras 24 horas após a injeção. Neste estudo, especialmente no grupo 3 em 1, o escore de dor foi adequado nesses pacientes.

A infusão contínua de bupivacaína a $0,125 \%$ a uma velocidade de $0,14 \mathrm{~mL} \cdot \mathrm{kg}^{-1} \cdot \mathrm{h}^{-1}$ é considerada boa escolha na manutenção do bloqueio "3 em 1" prolongado ${ }^{17}$. No tratamento da dor pós-operatória após a artroplastia total do joelho, a infusão contínua ou as técnicas controladas pelo paciente para o bloqueio "3 em 1" foram eficazes ${ }^{18}$. Entretanto, a técnica "3 em 1" leva à administração de grandes volumes de anestésico local com risco tóxico potencial causado pelo acúmulo do medicamento após períodos prolongados de infusão ${ }^{19}$

Edkin e col. ${ }^{20}$ mostraram que uma dose única no bloqueio femoral reduz a dor pós-operatória em pacientes com reconstrução do LCA. Neste estudo, 92\% dos pacientes não necessitaram de morfina por via venosa nas primeiras 24 horas. Em 1.200 casos consecutivos de procedimentos cirúrgicos complexos do joelho, William e col. ${ }^{21}$ mostraram que o uso do bloqueio dos nervos femoral-ciático foi associado com menos dor no pós-operatório antes da alta no dia do procedimento e com poucas internações hospitalares. O uso de bloqueios nervosos para o tratamento de dor aguda em pacientes submetidos à reconstrução do ligamento cruzado anterior está associado com a eliminação da necessidade de internação na unidade de recuperação pós-anestésica e alta hospitalar confiável no mesmo dia. Apesar da redução dos custos relacionados com a redução de pessoal, extrapolar os resultados para todos os tipos de procedimentos ortopédicos ambulatoriais invasivos pode indicar o potencial para criar uma economia hospitalar significativa ${ }^{22}$. Neste estudo, os escores de dor obtidos no grupo do bloqueio "3 em 1" foram menores do que os observados no grupo de analgesia contínua por via venosa $(p<0,001)$.

Em uma série de 211 cateteres de nervo femoral introduzidos usando-se técnica asséptica, Cuvillon e col. ${ }^{23}$ removeram os cateteres depois de 48 horas e realizaram cultura bacteriológica semiquantitativa da ponta de cada cateter. Nesse estudo, 57\% dos cateteres apresentaram colonização bacteriana. De acordo com esses resultados, nesta série os cateteres foram mantidos na bainha do nervo femoral apenas por 48 horas.

De acordo com o conceito da analgesia balanceada ${ }^{24}$, o ceterolaco por via venosa foi administrado a todos os pacientes antes do início da operação e a clonidina foi associada à infusão contínua do grupo de bloqueio "3 em 1". 
A adição de clonidina às soluções de anestésico local prolonga a duração da anestesia e analgesia após uma única injeção no bloqueio do plexo braquial ${ }^{25}$, mas não afeta a dor pós-operatória quando administrada como analgésico único ${ }^{26}$. O mecanismo de ação da clonidina nos nervos periféricos é desconhecido. Atualmente são propostos três mecanismos de ação: vasoconstrição local nos nervos periféricos pela ativação dos receptores adrenérgicos póssinápticos ${ }^{27}$, o que leva à redução na absorção sistêmica do anestésico local. Em segundo lugar, a clonidina poderia apresentar ação anestésica local. Sua aplicação tópica na córnea de coelhos tem ação anestésica de superfície 140 vezes maior do que a da procaína ${ }^{28}$. Essa hipótese ainda não foi comprovada in vivo ${ }^{27}$. Finalmente, a clonidina pode potencializar o efeito dos anestésicos locais ${ }^{29}$. Recentemente, Mannion e col. ${ }^{30}$ demonstraram que a clonidina, por via venosa, mas não por via peridural, prolongou a analgesia pósoperatória após o bloqueio do compartimento do psoas com levobupivacaína em pacientes com fratura de quadril. Apesar de toda essa controvérsia, a literatura indica evidências de que existe benefício na adição de baixas concentrações de clonidina aos anestésicos locais no tratamento da dor pósoperatória nos bloqueios nervosos contínuos ${ }^{2,14}$. Por essa razão, a clonidina na dose de $1 \mu \mathrm{g} \cdot \mathrm{mL}^{-1}$ foi adicionada à bupivacaína a $0,125 \%$ na infusão contínua do bloqueio "3 em 1". Neste estudo, não foram detectadas complicações atribuídas às técnicas de analgesia "3 em 1" ou sistêmica.

Concluindo, este estudo prospectivo, com distribuição aleatória, confirmou que o bloqueio "3 em 1" prolongado com bupivacaína e clonidina em sistemas de infusão contínua fornece analgesia mais eficaz após a reconstrução do LCA do joelho, maior satisfação do paciente, redução no consumo de morfina, com baixa incidência de NVPO quando comparado com a analgesia sistêmica.

\section{Extended Three-in-One Block versus Intravenous Analgesia for Postoperative Pain Management after Reconstruction of Anterior Cruciate Ligament of the Knee}

Víctor A. Contreras-Domínguez, M.D.; Paulina E. CarbonellBellolio, M.D.; Álvaro C. Ojeda-Greciet, M.D.; Edgardo S. Sanzana, M.D.

\section{INTRODUCTION}

Continuous femoral nerve block (CFB) has been used with good results as a postoperative analgesic technique in both hip and knee replacements ${ }^{1-3}$, in skin grafting in burned patients ${ }^{4}$, considering all the advantages and low side effects compared with other locoregional analgesic techniques or intravenous (IV) infusions $5,6$.
CFB is an excellent alternative to epidural analgesia (EA) or patient controlled analgesia (PCA) intravenous opioids in acute pain relief in major orthopedic surgery ${ }^{7}$. This technique also avoids complications such as epidural bleeding that can be produced in patients undergoing low molecular-weight heparin treatment ${ }^{8}$.

The continuous infusion of local anesthetics (LA) can produce systemic side effects such as confusion, hypotension, hypoxia, arrhythmias, convulsions and coma. However, with the use of CFB these complications are rare, due to the low concentrations of LA used and the low plasmatic level reached ${ }^{9}$.

During EA is frequent to have hypotension, cardiorespiratory depression or neurological symptoms, especially when used in older or high risk patients. The CFB seems to be very advantageous over EA ${ }^{10}$.

Postoperative pain after anterior cruciate ligament ( $A C L$ ) reconstruction is moderate to severe in $89 \%$ of the patients. The administration of preemptive analgesics (i.e. ketorolac $60 \mathrm{mg} \mathrm{IV}$ ) or intrarticular LA (bupivacaine $1 \mathrm{mg} \cdot \mathrm{kg}^{-1}$ ) in association with postoperative oral analgesia ${ }^{11}$ doesn't change pain scores.

The aim of this study was to clinically evaluate the efficacy of the continuous femoral block compared with intravenous analgesia in reconstruction of anterior cruciate knee ligament under arthroscopic assistance.

\section{METHODS}

After informed written consent and with institutional approval of the ethics committee, 60 ASA physical status I patients scheduled for elective unilateral reconstruction of anterior cruciate knee ligament (ACL), assisted by arthroscopy under spinal anesthesia were included in this study.

We included patients aged between 18 and 51 years old, with a body mass index $(\mathrm{BMI})<30$. Patients were excluded for the following reasons: if they had coagulation abnormality, LA allergy, local infection, preexisting neurological deficit, hypertension, diabetes, hepatic or kidney diseases, chronic treatment with alpha ${ }_{2}$-agonists (clonidine), or if they were unable to understand pain scales or the use of a PCA device. Patients were then randomly divided into two groups of 30 patients each, where Group 1 received a continuous infusion of bupivacaine $0.125 \%$ plus clonidine $1 \mu \mathrm{g} \cdot \mathrm{mL}^{-1}$ at the rate of $10 \mathrm{~mL} . \mathrm{h}^{-1}$ by a femoral catheter during postoperative period, and Group 2 received a continuous intravenous (IV) infusion of ketoprofen at a dose of $300 \mathrm{mg}$ in 24 hours.

In all patients of Group 1, 3-in-1 block was performed before spinal anesthesia by using the landmarks of Winnie et al. ${ }^{12}$. The femoral artery was located below the inguinal ligament, and an 18G short-beveled cannula (Polyplex ${ }^{\circledR}$ C 50, Polymedic, Carneres sur Seine, France) was inserted just lateral to the artery. The femoral nerve was accurately located with a peripheral nerve stimulator (TOF Watch $\mathrm{S}^{\circledR}$, Organon, Ireland). With a starting output of $1.5 \mathrm{~mA}$, the needle was 


\section{EXTENDED THREE-IN-ONE BLOCK VERSUS INTRAVENOUS ANALGESIA FOR POSTOPERATIVE PAIN MANAGEMENT AFTER RECONSTRUCTION OF ANTERIOR CRUCIATE LIGAMENT OF THE KNEE}

advanced under intermittent aspiration at an angle of $30^{\circ}$ to $45^{\circ}$ to the skin until twitches of the quadriceps muscle (ascension of the patella) were elicited.

Its position was then optimized and judged adequate when output lower than $0.4 \mathrm{~mA}$ still elicited contraction of the quadriceps. A $20 \mathrm{G}$ catheter was then inserted using a Seldinger technique and threaded $12 \mathrm{~cm}$ into the femoral nerve sheath. After a negative aspiration test for blood, the catheter was fixed.

All patients of both groups received spinal anesthesia. Lumbar puncture was performed at $L_{3}-L_{4}$ or $L_{4}-L_{5}$ interspace with a $25 \mathrm{G}$ pencil point needle and $12.5 \mathrm{mg}$ of $0.75 \%$ hyperbaric bupivacaine with $2.5 \mu \mathrm{g}$ sufentanyl were administered. In all patients sedation during surgery was provided by a target-controlled-infusion ( $\mathrm{TCl}$ ) of propofol 1\% (Base Primea Orchestra ${ }^{\circledR}$, Fressenius-Vial, France). As well, all patients received $30 \mathrm{mg}$ of intravenous ketorolac before pneumatic ischemia was started, and then, three times daily during the first 48 hours postoperatively.

In the recovery room, the correct positioning of the femoral nerve sheath catheter was confirmed by an injection of 10 $\mathrm{mL}$ bupivacaine $0.125 \%$ plus $1 \mu \mathrm{g} \cdot \mathrm{mL}^{-1}$ of clonidine. Loss of temperature sensation (sensory block) was assessed by using an ether-soaked swab involving the distribution of the femoral nerve (anterior aspect of the thigh). After this test, the PMP pump (Abbott Laboratories, USA) was started with a solution of bupivacaine $0.125 \%$ plus clonidine $1 \mu \mathrm{g} \cdot \mathrm{mL}^{-1}$ at the rate of $10 \mathrm{~mL} \cdot \mathrm{h}^{-1}$ (Group 1). Patients in Group 2 received IV continuous analgesia with $300 \mathrm{mg}$ of ketoprofen in 24 hours. Supplemental analgesia was standardized by the administration of IV PCA morphine (bolus $1.5 \mathrm{mg}$, lockout time 10 minutes, security range 20 $\mathrm{mg}$ in 4 hours).

Immediately after recovery room discharge, all patients started identical pharmacological therapy (antibiotics, low molecular-weight heparin) and kinetic assistance with a passive motion machine that was applied 8 hours after discharge. Pain was measured by visual analog scale (VAS, ranging from $0=$ no pain to $100=$ worst pain imaginable) and recorded at 2, 6, 12, 24, 36 and 48 hours after surgery.
Side effects, morphine consumption and satisfactory scores (by using a VAS rating from $0=$ not satisfied to $100=$ entirely satisfied) at the end of the study period were recorded.

All data were collected by other anesthesiologists not involved in the anesthetic procedure or in the patient care in the recovery room.

A power analysis suggested that 15 patients would need to be enrolled in the study groups to observe at least $50 \%$ reduction in the VAS scores between the IV continuous analgesia and the femoral block group to provide a 95\% chance of detecting such a reduction at the 0.01 level of significance. To improve the clinical significance of our results, we decided to include 30 patients in each group. Statistical analysis was performed by using a one-way analysis of variance followed by a Bonferroni t-test, for the parametric data. The PCA morphine demands were analyzed with a KruskalWallis test. Demographic data were compared by using Chisquare test. Values of $p<0.05$ were considered significant. Results are expressed as mean \pm SD.

\section{RESULTS}

Demographic data are presented in table I. No difference was noted among the groups. In the recovery room, complete loss of temperature sense in the distribution of the femoral nerve was observed in all patients of Group 1.

The VAS Scores at 2, 4, 6, 24, 36 and 48 postoperative hours, morphine consumption, incidence of nausea and vomiting (PONV) and satisfaction score at $48 \mathrm{~h}$ are presented in table II. Pain score at 4, 6, 24, 36 and 48 hours were significantly lower in Group 1 compared with Group 2 ( $p<0.001$ and $p<0.01)$. When compared with Group 1, morphine consumption was significantly higher in Group 2 ( $p<0.001)$.

The satisfaction Score was lower than 60 points in Group 2, and higher than 80 points in Group 1 ( $p<0.05)$.

The incidence of PONV in Group 1 was significantly lower than Group 2 ( $p<0.01)$.

In Group 1 femoral nerve block was well maintained during the entire study period. Except for PONV, no other side effects or technical problems were noted in the two groups.

Table I - Demographic Data

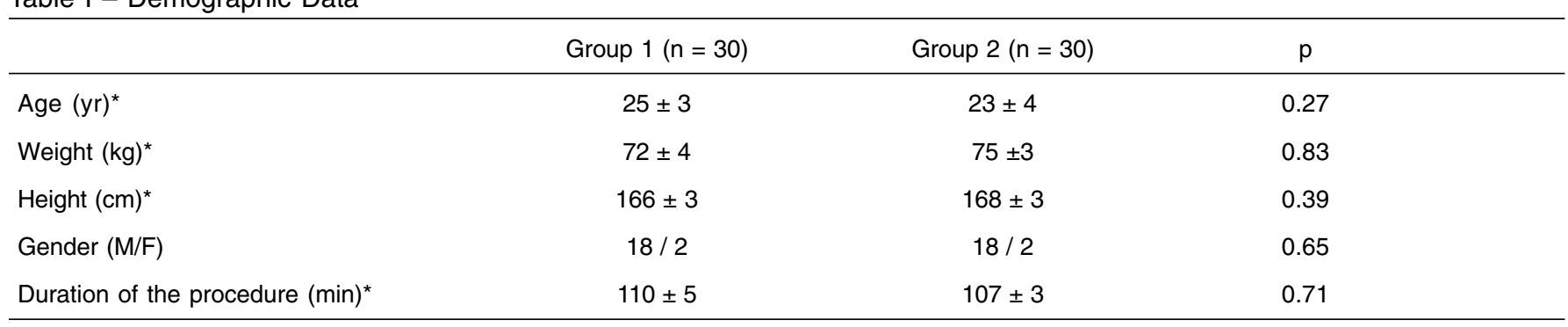

Group 1 - continuous femoral nerve block (three-in-one); Group 2 - continuous intravenous analgesia.

* Values are expressed as Mean \pm SD.

Statistical analysis was done with Analysis of Variance or Chi-square. 
Table II - Pain Scores, Morphine Consumption, Postoperative Nausea and Vomiting and Satisfaction Score in the Two Groups

\begin{tabular}{lccc}
\hline & Group $1(\mathrm{n}=30)$ & Group 2 $(\mathrm{n}=30)$ & $\mathrm{p}$ \\
\hline VAS $(\mathrm{mm})^{*}$ & & & \\
$2 \mathrm{~h}$ & $18(10-40)$ & $20(15-25)$ & 0.26 \\
$4 \mathrm{~h}$ & $20(15-20)$ & $45(30-60)$ & 0.001 \\
$6 \mathrm{~h}$ & $25(10-40)$ & $50(30-60)$ & 0.001 \\
$24 \mathrm{~h}$ & $19(10-30)$ & $40(30-60)$ & 0.001 \\
$36 \mathrm{~h}$ & $12(10-20)$ & $20(15-20)$ & 0.01 \\
$48 \mathrm{~h}$ & $14(10-22)$ & $21(14-22)$ & 0.01 \\
Morphine (mg / 48 hr) \# & $4.5 \pm 1.5$ & $25.5 \pm 3.0$ & 0.001 \\
PONV (\%) \#\# & 6.7 & 20 & 0.01 \\
Satisfaction Score \#\#\# & $87 \pm 2$ & $57 \pm 5$ & 0.05 \\
\hline
\end{tabular}

Group 1 - continuous femoral (three-in-one) block; Group 2 - continuous intravenous analgesia; VAS - Visual Analog Scale; PONV postoperative nausea and vomiting.

* Expressed as Median (25 $5^{\text {th }}-75^{\text {th }}$ Percentiles).

\# Values expressed as Means \pm SD.

\#\# Expressed as percentages.

\#\# Expressed as ratio (VAS rating from $0=$ not satisfied to $100=$ entirely satisfied).

\section{DISCUSSION}

This prospective, randomized trial showed that femoral nerve block with bupivacaine and clonidine in continuous infusion delivery system provides more efficient analgesia, highest patient satisfaction, lowest pain scores and consumption of intravenous morphine and lower incidence of PONV than intravenous analgesia.

The reconstruction of ACL based on "bone-tendon-bone" technique (autologous grafting of patellar tendon) is highly recommended in young sportsman patients because of its major biomechanical resistance and good functional results observed in long term series.

The management of postoperative pain after ACL reconstruction has been developed by the intravenous analgesia (continuous or PCA techniques), intra-articular analgesia (IA), epidural analgesia (EA) and femoral nerve block (threein-one block), either with single shot or with continuous administration.

The IV analgesia in patients undergoing either general or regional anesthesia is commonly associated with high consumption of intravenous morphine, high incidence of nausea and postoperative vomiting (PONV) and low patient's satisfaction scores ${ }^{13}$. This is corroborated in this series of 60 patients, where IV analgesia group has higher pain scores, morphine consumption, PONV incidence and less satisfaction scores compared to the "three-in-one" block group $(p<0.001)$. The role of other analgesic intravenous drugs may be interesting in the management of patients undergoing orthopedic surgery under general anesthesia. The administration of small doses of ketamine in the perioperative period reduces postoperative morphine requirements and improves mobilization 24 hours after arthroscopic anterior ligament repair ${ }^{13,14}$.
This interesting strategy could be important to be used in association with intravenous analgesia. In this experience, the postoperative morphine consumption and the incidence of PONV were significantly higher in the intravenous Group than in the "three-in-one" block Group.

The epidural analgesia provides good postoperative analgesia, but increases the incidence of adverse effects such as hypotension, bilateral motor and sensory block and urinary retention. Problems associate with the management of the epidural catheter and the risk of epidural hematoma in patients undergoing anticoagulant therapy reduces its use ${ }^{8}$. Chew et al. ${ }^{15}$ demonstrated the utility of infrapatelar compartment continuous infusion of bupivacaine 0.25 or $0.5 \%$. Branson et al. ${ }^{16}$ used diluted intrarticular (IA) morphine (2 or $5 \mathrm{mg}$ ) with good analgesic results in the first 24 hours. After this period, the pain increased and the kinetic therapy resulted difficult. The IA technique is limited to management of postoperative pain in major knee surgery because its utility is restricted to the first 24 hours after injection. In this study, especially in the three-in-one group, the pain scores were adequate for these patients.

The continuous infusion of $0.125 \%$ of bupivacaine at the rate of $0.14 \mathrm{~mL} . \mathrm{kg}^{-1} \cdot \mathrm{h}^{-1}$ is considered a very good choice to maintain the extended "three-in-one" block ${ }^{17}$. In the management of postoperative pain after total knee arthroplasty, the continuous infusion or patient-controlled techniques for "three-inone" block were efficient ${ }^{18}$. However, the "three-in-one" technique leads to the administration of large volumes of local anesthetic with a potential risk of toxicity caused by accumulation of the drug after prolonged periods of infusion ${ }^{19}$.

Edkin et al. ${ }^{20}$ showed the utility of a single-shot dose in femoral block to reduce the postoperative pain in patients with ACL reconstruction. In this study, $92 \%$ of the patients did not 


\section{EXTENDED THREE-IN-ONE BLOCK VERSUS INTRAVENOUS ANALGESIA FOR POSTOPERATIVE PAIN MANAGEMENT AFTER RECONSTRUCTION OF ANTERIOR CRUCIATE LIGAMENT OF THE KNEE}

require supplemental IV morphine during the first 24 hours. In 1.200 consecutive cases of complex surgery of knee, William et al. ${ }^{21}$ showed that the use of femoral-sciatic nerve blocks for complex outpatients' knee surgery was associated with less postoperative pain before same-day discharge and with few hospital admissions. The use of nerve blocks for acute pain management in patients undergoing anterior cruciate ligament reconstruction is associated with post anesthesia care unit (PACU) bypass and reliable same-day discharge. Although the cost savings via staffing reductions, extrapolating the results to a large volume of all type of invasive outpatients orthopedic procedures may have the potential to create significant hospital cost saving ${ }^{22}$. In this study, the pain scores obtained in the "three-in-one" block Group were lower than those obtained by the intravenous continuous analgesia Group $(p<0.001)$.

In a series of 211 femoral nerve catheters that were placed using aseptic technique, Cuvillon et al. ${ }^{23}$ removed the catheters after 48 hours and semi-quantitative bacteriological culture was performed on each distal catheter tip. In this study, $57 \%$ of catheters had positive bacterial colonization. Accordingly to these results, in this series was maintained the femoral nerve sheath catheters in place only for the first 48 postoperative hours.

In accordance with the concept of balanced analgesia ${ }^{24}$, IV ketorolac was administrated to all patients before starting surgery, and clonidine was added in continuous infusion "three-in-one" block Group.

The addition of clonidine to local anesthetic solutions prolongs duration of anesthesia and analgesia after a singleshot brachial plexus block ${ }^{25}$, but does not affect postoperative pain when given as a sole analgesic ${ }^{26}$. The mechanism of action of clonidine on peripheral nerves is unknown. Actually, three mechanisms of action may be proposed: local vasoconstriction of peripheral nerves by activation of postsynaptic adrenergic receptors ${ }^{27}$ which lead to a decrease in the systemic absorption of the local anesthetic. In second place, clonidine may have a local anesthetic activity. Its topic application on the rabbit cornea results in 140 times more potent as a surface anesthetic compared to procaine ${ }^{28}$. In vivo, this hypothesis has not yet been proved ${ }^{27}$. Finally, clonidine may have a potentiating effect on local anesthetics ${ }^{29}$. Recently, Mannion et al. ${ }^{30}$ demonstrated that intravenous but not perineural clonidine prolongs postoperative analgesia after psoas compartment block with levobupivacaine in patients with hip fractures. Even though all this controversies, literature evidences a benefit in the addition of clonidine to low concentrations of local anesthetic solutions for postoperative pain management in continuous peripheral nerve blocks ${ }^{2,14}$. For this reason, was added clonidine in a concentration dose of $1 \mu \mathrm{g} \cdot \mathrm{mL}^{-1}$ to bupivacaine $0.125 \%$ in "threein-one" block continuous infusion.

In this study, there were no complications attributed to the use of "three-in-one" block or intravenous analgesia techniques.
In conclusion, this prospective, randomized trial confirms that extended "three-in-one" block with bupivacaine and clonidine in continuous infusion delivery system provides more efficient analgesia after reconstruction of ACL of the knee, better patient satisfaction scores and less consumption of intravenous morphine, with low incidence of PONV compared with intravenous analgesia.

\section{REFERÊNCIAS - REFERENCES}

01. Singelyn FJ, Vanderest PE, Gouverneur JM - Extended femoral nerve sheath block after total hip arthroplasty: continuous versus patient-controlled techniques. Anesth Analg, 2001; 92:455-459.

02. Singelyn FJ, Deyaert M, Joris D et al. - Effects of intravenous patient-controlled analgesia with morphine, continuous epidural analgesia, and continuous three-in-one block on postoperative pain and knee rehabilitation after unilateral total knee arthroplasty. Anesth Analg, 1998;87:88-92.

03. Enneking FK, Wedel DJ - The art and science of peripheral nerve blocks. Anesth Analg, 2000;90:1-2.

04. Cuignet O, Pirson J, Boughrouph J et al. - The efficacy of continuous fascia iliaca compartment block for pain management in burn patients undergoing skin grafting procedures. Anesth Analg, 2004;98:1077-1081.

05. Singelyn FJ, Contreras V, Gouverneur JM - Epidural anesthesia complicating continuous 3-in-1 lumbar plexus block. Anesthesiology, 1995;83:217-220.

06. Adam F, Jaziri S, Chauvin M - Psoas abscess complicating femoral nerve block catheter. Anesthesiology, 2003;99:230-231.

07. Chelly JE, Greger J, Gebhard R et al. - Continuous femoral blocks improve recovery and outcome of patients undergoing total knee arthroplasty. J Arthroplasty, 2001;16:436-45.

08. Auroy $Y$, Benhamou D, Bargues $L$ et al. - Major complications of regional anesthesia in France: the SOS Regional Anesthesia Hotline Service. Anesthesiology, 2002;97:1274-1280.

09. Chelly JE - Complications of Continuous Peripheral Nerve Blocks, em: Chelly JE, Casati A, Fanelli G — Continuous Peripheral Nerve Block Techniques. Philadelphia, Mosby, 2001;21-5.

10. Ben-David B, Chelly JE - Continuous peripheral neural blockade for postoperative analgesia: practical advantages. Anesth Analg, 2003;96:1537-1538.

11. Brown DW, Curry CM, Ruterbories LM et al. - Evaluation of pain after arthroscopically assisted anterior cruciate ligament reconstruction. Am J Sports Med, 1997;25:182-186.

12. Winnie AP, Ramamurthy S, Durrani $Z-$ The inguinal paravascular technique of lumbar plexus anesthesia: the "3-in-1" block. Anesth Analg, 1973;52:989-996.

13. Menigaux C, Fletcher D, Dupont $X$ et al. - The benefits of intraoperative small-dose ketamine on postoperative pain after anterior cruciate ligament repair. Anesth Analg, 2000;90:129-135.

14. Menigaux C, Guignard B, Fletcher D et al. — Intraoperative smalldose ketamine enhances analgesia after outpatient knee arthroscopy. Anesth Analg, 2001;93:606-612.

15. Chew HF, Evans NA, Stanish WD - Patient-controlled bupivacaine infusion into the infrapatellar fat pad after anterior cruciate ligament reconstruction. Arthroscopy, 2003;19:500-505.

16. Brandsson S, Karlsson J, Morberg $P$ et al. - Intraarticular morphine after arthroscopic ACL reconstruction: a double-blind placebo controlled study of 40 patients. Acta Orthop Scand, 2000;71:280-285.

17. Anker-Moller E, Spangsberg N, Dahl JB et al. - Continuous blockade of the lumbar plexus after knee surgery: a comparison of 
the plasma concentrations and analgesic effect of bupivacaine $0.250 \%$ and $0.125 \%$. Acta Anaesthesiol Scand, 1990;34:468472.

18. Singelyn FJ, Gouverneur JM - Extended three-in-one block after total knee arthroplasty: continuous versus patient-controlled techniques. Anesth Analg, 2000;91:176-180.

19. Esteve M, Veillette $Y$, Ecoffey $C$ et al. - Continuous block of the femoral nerve after surgery of the knee: pharmacokinetics of bupivacaine. Ann Fr Anesth Réanim, 1990;9:322-325.

20. Edkin BS, Spindler KP, Flanagan JF - Femoral nerve block as an alternative to parenteral narcotics for pain control after anterior cruciate ligament reconstruction. Arthroscopy, 1995;11:404-409.

21. Williams BA, Kentor ML, Vogt MT et al. - Femoral-sciatic nerve blocks for complex outpatient knee surgery are associated with less postoperative pain before same-day discharge: a review of 1,200 consecutive cases from the period 1996-1999. Anesthesiology, 2003;98:1206-1213.

22. Williams BA, Kentor ML, Vogt MT et al. - Economics of nerve block pain management after anterior cruciate ligament reconstruction. Potential hospital cost savings via associated postanesthesia care unit bypass and same day discharge. Anesthesiology, 2004;100:697-706.

23. Cuvillon P, Ripart J, Lalourcey $L$ et al. - The continuous femoral nerve block catheter for postoperative analgesia: bacterial colonization, infectious rate and adverse effects. Anesth Analg, 2001;93:1045-1049.

24. Kehlet $\mathrm{H}-$ Surgical stress: the role of pain and analgesia. $\mathrm{Br} \mathrm{J}$ Anaesth, 1989;63:189-195.

25. Singelyn FJ, Gouverneur JM, Robert A - A minimum dose of clonidine added to mepivacaine prolongs the duration of anesthesia and analgesia after axillary brachial plexus block. Anesth Analg, 1996;83:1046-1050.

26. Sia S, Lepri A - Clonidine administered as an axillary block does not affect postoperative pain when gives as the sole analgesic. Anesth Analg, 1999;88:1109-12.

27. Langer SZ, Duval N, Massingham R - Pharmacologic and therapeutics significance of alpha-adrenoreceptor subtypes. $J$ Cardiovasc Pharmacol, 1985;7(suppl 8):s1-8.

28. Starke K, Wagner J, Schümann HJ - Adrenergic neuron blockade by clonidine: comparison with guanethidine and local anesthetics. Arch Int Pharmacodyn Ther, 1972;195:291-308.

29. Gaumann DM, Brunet PC, Jirounek $P$ - Clonidine enhances the effects of lidocaine on $\mathrm{c}$-fiber action potential. Anesth Analg, 1992;74:719-725.

30. Mannion S, Hayes I, Loughnane F et al. - Intravenous but not perineural clonidine prolongs postoperative analgesia after psoas compartment block with $0.5 \%$ levobupivacaine for hip fracture surgery. Anesth Analg, 2005;100:873-878.

\section{RESUMEN}

Contreras-Domínguez VA, Carbonell-Bellolio PE, Ojeda-Greciet AC, Sanzana ES - Bloqueo 3 en 1 Prolongado versus Analgesia Sistemica en el Tratamiento del Dolor Postoperatorio Después de la Reconstrucción del Ligamento Cruzado Anterior de la Rodilla.

JUSTIFICATIVA Y OBJETIVOS: El bloqueo femoral continuo (tresen-uno) se usa para la analgesia postoperatoria de artroplastia de cadera y rodilla con buenos resultados, presentando ventajas sobre otras técnicas de analgesia loco regional o sistemica y con baja incidencia de complicaciones. El objetivo de este estudio fue el de evaluar clínicamente la utilidad del bloqueo femoral continuo en comparación con la analgesia intravenosa en la reconstrucción del ligamento cruzado anterior.

MÉTODOS: Se realizó un estudio prospectivo controlado con 60 pacientes de estado físico ASA I. Los pacientes fueron divididos en dos grupos: Grupo 1 ( $n=30)$ : bloqueo femoral continuo con infusión de bupivacaína y clonidina; y Grupo $2(n=30)$ : infusión intravenosa de cetoprofeno. La intervención quirúrgica se hizo bajo raquianestesia y sedación. El tratamiento del dolor postoperatorio se hizo con analgesia controlada por el paciente (PCA) usando morfina. El dolor postoperatorio fue registrada 2, 4, 6, 24 y 36 horas después de la intervención quirúrgica usando la Escala Visual Analógica (VAS). El consumo de morfina, la satisfacción de los pacientes y las complicaciones también fueron registrados.

RESULTADOS: En el Grupo 1, el VAS postoperatorio entre 4 y 48 horas trás de la intervención quirúrgica fue de $21 \mathrm{~mm} \pm 2$ y en el Grupo 2 fue de $45 \mathrm{~mm} \pm 4$ ( $p<0.001)$. El uso de morfina en el período de 4 a 48 horas en el Grupo 1 fue de $4,5 \mathrm{mg} \pm 1,5$ y en el Grupo 2 de 25,5 mg \pm 3 ( $p<0.001)$. En el Grupo 1, 6,7\% de los pacientes presentaron náusea o vómito en el postoperatorio (NVPO) comparado con $20 \%$ del Grupo 2.

CONCLUSIONES: El bloqueo 3-en-1 con bupivacaína y clonidina en infusión continua suministró analgesia más eficaz, mayor satisfacción del paciente, redució el consumo de morfina intravenosa y presentó una menor incidencia de NVPO que la analgesia sistemica. 